\title{
Terahertz pulse-driven collective mode in the nematic superconducting state of $\mathrm{Ba}_{1-x} \mathrm{~K}_{x} \mathrm{Fe}_{2} \mathrm{As}_{2}$
}

\author{
Romain Grasset $\mathbb{D}^{1,2,3 凶}$, Kota Katsumi ${ }^{2}$, Pierre Massat ${ }^{4}$, Hai-Hu Wen ${ }^{5}$, Xian-Hui Chen $\mathbb{1}^{6}$, Yann Gallais $\mathbb{D}^{1,2,4 凶}$ and Ryo Shimano ${ }^{1,2 凶}$
}

We investigate the collective mode response of the iron-based superconductor $\mathrm{Ba}_{1-}{ }_{-} \mathrm{K}_{x} \mathrm{Fe}_{2} \mathrm{As}_{2}$ using intense terahertz (THz) light. In the superconducting state a $\mathrm{THz}$ Kerr signal is observed and assigned to nonlinear $\mathrm{THz}$ coupling to superconducting degrees of freedom. The polarization dependence of the $\mathrm{THz}$ Kerr signal is remarkably sensitive to the coexistence of a nematic order. In the absence of nematic order the $\mathrm{C}_{4}$ symmetric polarization dependence of the THz Kerr signal is consistent with a coupling to the Higgs amplitude mode of the superconducting condensate. In the coexisting nematic and superconducting state the signal becomes purely nematic with a vanishing $C_{4}$ symmetric component, signaling the emergence of a superconducting collective mode activated by nematicity.

npj Quantum Materials (2022)7:4; https://doi.org/10.1038/s41535-021-00411-9

\section{INTRODUCTION}

Superconductivity with coexisting electronic orders can be found in various strongly correlated systems. Among these orders electron nematicy, where the electron fluid breaks the discrete rotational symmetry of the underlying lattice, has recently emerged as an ubiquitous phase in many superconductors ranging from cuprates ${ }^{1}$, to iron-based superconductors ${ }^{2}$ where superconductivity emerges within a nematic phase, and more recently doped $\mathrm{Bi}_{2} \mathrm{Se}_{3}{ }^{3}$ and twisted bi-layer graphene ${ }^{4,5}$ where the superconductivity itself may have a nematic component. In iron-based superconductors (Fe SC), superconductivity is found to coexist with both stripe-like magnetic spin-density-wave (SDW) and nematic orders. $\mathrm{BaFe}_{2} \mathrm{As}_{2}$, a member of this family, undergoes a nematicstructural transition from a $C_{4}$ to a $C_{2}$ symmetric phase, followed by a SDW transition ${ }^{2,6}$. The $C_{4}$ rotational symmetry breaking is triggered by electronic degrees of freedom and has been dubbed nematic for this reason ${ }^{2,7,8}$. With increasing doping by substitution (e.g., Ba with $\mathrm{K}$ or Fe with $\mathrm{Co}^{6,9}$ ), the $\mathrm{C}_{2}$ symmetric nematic-SDW phase, hereafter called the $C_{2}$ phase, is weakened and a superconducting (SC) dome forms around a possible quantum critical point. The coexistence with the $C_{2}$ phase can profoundly impact the nature of SC order, by coupling different nearly degenerate pairing channels like $s$ and $d$-wave ${ }^{10,11}$, or inducing an orbitally-selective SC state stin $^{12,13}$.

One way to gain insight into the coupling between nematic and SC degrees of freedom is to study the collective modes of the SC state upon entering the $C_{2}$ SC phase. Theoretically, intertwined electronic orders where superconductivity coexists with other electronic orders can lead to a rich spectrum of SC collective modes ${ }^{14-24}$. In a single band conventional superconductor the collective mode excitation spectrum consists of two modes: the Nambu-Goldstone phase mode which is shifted to the plasma frequency through the Anderson-Higgs mechanism, and the Higgs amplitude mode located at twice the SC gap energy. The Higgs mode does not couple linearly to light ${ }^{14,25,26}$. Except in very special cases like charge-density-wave superconductors ${ }^{27-30}$, its observation has remained elusive until very recently. In this context, strong terahertz $(\mathrm{THz})$ pulses have emerged as a tool of choice because they can access hidden SC collective modes via nonlinear optical processes ${ }^{31-37}$. This has led to the observation of the SC Higgs mode in several SC materials like $\mathrm{NbN}$ and $\mathrm{Nb}_{3} \mathrm{Sn}$, but also in cuprates and $\mathrm{Fe} \mathrm{SC}^{38-45}$. In the case of Fe SC however, little is known experimentally about the impact of nematicity on SC collective modes like the Higgs.

Here we investigate the $\mathrm{THz}$ nonlinear response of the $\mathrm{Fe} \mathrm{SC}$ $\mathrm{Ba}_{1-x} \mathrm{~K}_{x} \mathrm{Fe}_{2} \mathrm{As}_{2}$ where superconductivity coexists with a nematic order using a THz pump near-infrared (NIR) probe scheme. In the SC state, we observe an instantaneous response which follows the square of the $\mathrm{THz}$ electric field which is assigned to the nonlinear $\mathrm{THz}$ Kerr effect. In the absence of a coexisting nematic order the $\mathrm{THz}$ Kerr signal displays a $\mathrm{C}_{4}$ symmetric polarization dependence consistent with a nonlinear coupling to the SC Higgs mode. In the presence of a coexisting nematic order, the THz Kerr signal displays a drastic change in its polarization dependence: from fully symmetric in the $C_{4}$ symmetric SC phase to fully nematic in the $C_{2}$ symmetric phase. We show theoretically that the onset of the THz Kerr Higgs response in the nematic channel can be qualitatively explained by taking into account the anisotropy of the electronic structure in the $\mathrm{C}_{2}$ nematic phase. However, the complete disappearance of the $\mathrm{C}_{4}$ symmetric signal in the $C_{2}$ SC phase cannot be captured within this simple picture, indicating a non-trivial interplay between the nematic and superconducting order parameters and the emergence of a collective mode, distinct from the Higgs mode. We tentatively assigned this mode to the Bardasis-Schrieffer (BS) mode connecting s-wave and d-wave superconducting ground states which become mixed in the $C_{2}$ symmetric SC phase.

\section{RESULTS}

\section{Non-linear THz Kerr effect}

We studied two single crystals of $\mathrm{Ba}_{1-x} \mathrm{~K}_{x} \mathrm{Fe}_{2} \mathrm{As}_{2}$ with $T_{\mathrm{c}}=26 \mathrm{~K}$ (UD26) and $T_{\mathrm{c}}=37 \mathrm{~K}$ (UD37). The UD26 crystal is slightly

\footnotetext{
${ }^{1}$ Cryogenic Research Center, The University of Tokyo, Tokyo 113-0032, Japan. ${ }^{2}$ Department of Physics, The University of Tokyo, Tokyo $113-0033$, Japan. ${ }^{3}$ Laboratoire des Solides Irradiés, Ecole Polytechnique, CNRS, CEA, Institut Polytechnique de Paris, F-91128 Palaiseau, France. ${ }^{4}$ Université de Paris, CNRS, Laboratoire Matériaux et Phénomènes Quantiques, Paris 75013, France. ${ }^{5}$ National Laboratory of Solid State Microstructures and Department of Physics, Nanjing University, Nanjing 210093 , China. ${ }^{6}$ Hefei National Laboratory for Physical Sciences at Microscale and Department of Physics, and CAS Key Laboratory of Strongly-Coupled Quantum Matter Physics, University of Science and Technology of China, Hefei 230026 Anhui, China. ${ }^{\bowtie}$ email: romain.grasset@polytechnique.edu; yann.gallais@u-paris.fr; shimano@phys.s.u-tokyo.ac.jp
} 


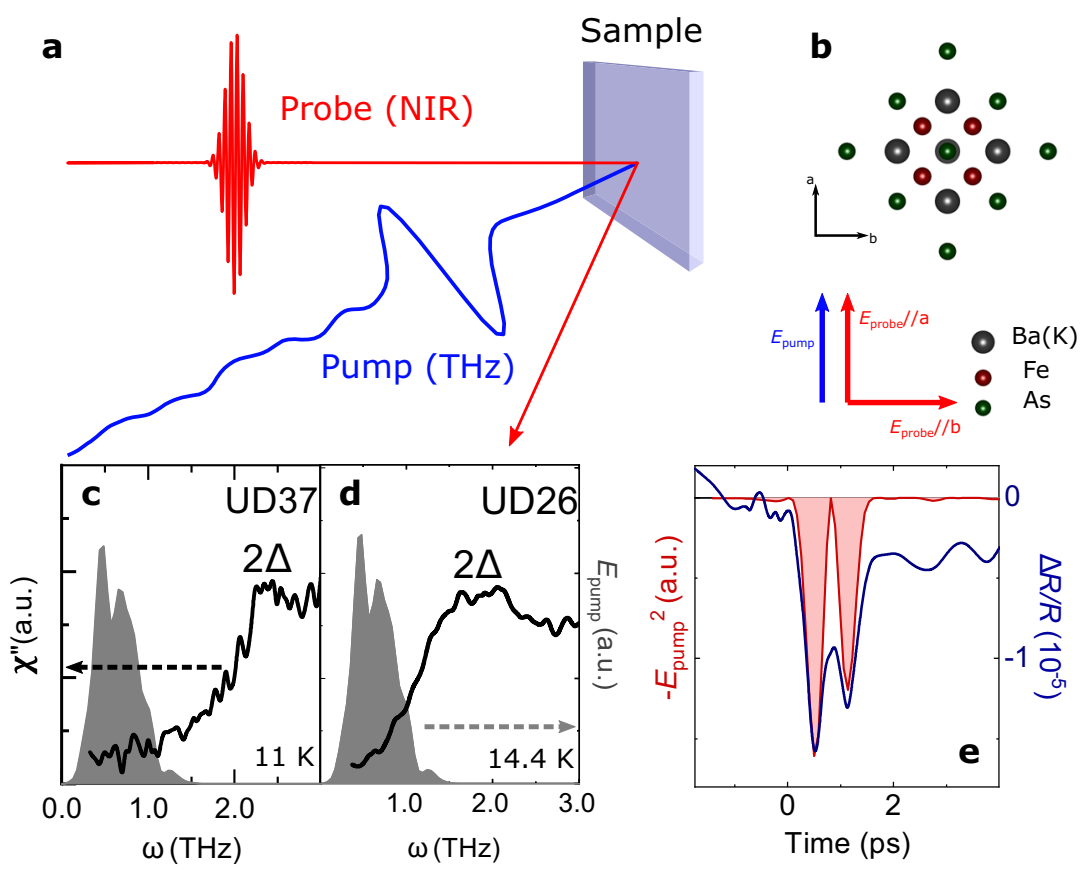

Fig. 1 Nonlinear THz response in $\mathbf{B a}_{1-x} \mathbf{K}_{\mathbf{x}} \mathrm{Fe}_{\mathbf{2}} \mathbf{A s}_{2}$. a Sketch of the THz pump near-infrared (NIR) probe measurements. $\mathbf{b}$ Crystal structure of $\mathrm{BaFe}_{2} \mathrm{As}_{2}$ and the two polarization configurations used to determine the $\mathrm{C}_{4}$ symmetric and nematic components of the transient reflectivity. Raman spectra of $\mathrm{Ba}_{1-x} \mathrm{~K}_{x} \mathrm{Fe}_{2} \mathrm{As}_{2}$ in the $\mathrm{B}_{1 \mathrm{~g}}$ symmetry for UD37 (c) and UD26 (d) below $T_{c}$. $2 \Delta$ indicates superconducting gap from the hole pockets. The gray curve represents the energy spectrum of the $\mathrm{THz}$ pump. e For UD37, $\Delta R / R$ (blue line) along one of the Fe-Fe axis at $20 \mathrm{~K}$ $\left(T<T_{\mathrm{c}}\right)$ as a function of delay time between the pump and probe pulses. The instantaneous component that follows $E_{\text {pump }}^{2}$ (red line) component corresponds to the THz Kerr effect.

underdoped and exhibits a simultaneous nematic/SDW transition at $T_{\mathrm{N}} \sim T_{\mathrm{S}} \sim 90 \mathrm{~K}$. The UD37 crystal only exhibits a superconducting transition and is close to optimal doping. The terahertz-pump optical reflectivity probe (TPOP) measurement scheme is depicted in Fig. 1a. Measurements were carried out with a fixed $\mathrm{THz}$ pump polarization along the Fe-Fe direction but two different probe polarizations either parallel or perpendicular to the pump polarization (Fig. 1b). Fe-Fe directions are identified by a $45^{\circ}$ tilt with respect to the edges of the crystals which are square-shaped.

In Fig. 1c, d, we compare the THz pump spectrum with the SC state Raman spectra of the two samples (See Supplementary Note 1 for more details). With an energy centered around $\omega_{p}=0.6$ $\mathrm{THz}=20 \mathrm{~cm}^{-1}$, the $\mathrm{THz}$ pump spectrum is located below the lowest superconducting gap $2 \Delta_{\mathrm{h}}$ observed by Raman scattering. Based on previous Raman and angle-resolved photoemission spectroscopy measurements this gap is assigned to the $\Gamma$ centered hole pockets. The TPOP signal $\frac{\Delta R}{R}$ of UD37 below $T_{\mathrm{C}}$ is shown in Fig. 1e. It consists of essentially two components, an instantaneous component that follows the square of the $\mathrm{THz}$ electric field (E-field) (red line in Fig. 1e) and a broader decaying component which last several picoseconds after the pump pulse. In the following we will mostly focus on the instantaneous component, the $\mathrm{THz}$ Kerr effect, where the strong $\mathrm{THz} E$-field modulates the optical reflectivity in the NIR regime ${ }^{46}$. We note that in our measurements we only detect an instantaneous component that is proportional to the square of the $\mathrm{THz} E$-field, consistent with the centrosymmetric crystal structure of $\mathrm{Ba}_{1-x} \mathrm{~K}_{x} \mathrm{Fe}_{2} \mathrm{As}_{2}$. No forbidden odd contribution is observed, as recently reported in the SC state of $\mathrm{Nb}_{3} \mathrm{Sn}^{47}$ and attributed to $\mathrm{THz}$ field symmetry breaking.

The THz Kerr signal is described by a third-order nonlinear susceptibility $x^{(3)}(\omega ; \omega,+\Omega,-\Omega)^{48,49}$, where $\omega$ and $\Omega$ are the frequencies of the NIR pulse and THz-pump pulse, respectively. The $\mathrm{THz}$ pulse-induced reflectivity change $\Delta R / R$ can be expressed in terms of $X^{(3)}$ (see Supplementary Note 3) as:

$$
\frac{\Delta R}{R}\left(E_{i}^{\text {probe }}, E_{j}^{\text {probe }}\right) \sim \frac{1}{R} \frac{\delta R}{\delta \epsilon_{1}} \epsilon_{0}\left[\operatorname{ReX}_{i j k l}^{(3)}\right] E_{k}^{\text {pump }} E_{l}^{\text {pump }}
$$

where $E_{i}$ denotes the $i$ th component of the THz-pump or probe $E$-field and $\epsilon_{1}$ is the real part of the dielectric constant at $1.5 \mathrm{eV}$. The instantaneous Kerr signal of interest here implies $\Omega=0$ in $\chi^{(3)}$. It is therefore independent of the pump frequency $\Omega$ and nonresonant ${ }^{42,49}$. This is in contrast with the third-harmonic generation (THG) signal which is resonant when the pump frequency $\Omega$ equals the superconducting gap $\Delta^{31}$.

In general, the onset of a THz Kerr signal below $T_{\mathrm{c}}$ can be assigned to two different processes: coupling to charge density fluctuations (CDF) like the one observed in Raman experiments, or to the SC Higgs mode. As previously shown in the case of $\mathrm{NbN}$ and cuprates important clues about the origin of the $\mathrm{THz}$ Kerr signal, and other third-order nonlinear effects like THG, can be obtained by investigating its polarization dependence $32,35,36,40,42,43$.

Assuming $C_{4}$ tetragonal symmetry for the normal state of $\mathrm{Ba}_{1-x} \mathrm{~K}_{x} \mathrm{Fe}_{2} \mathrm{As}_{2}$, we can analyze the polarization dependence of $X^{(3)}\left(\theta_{\text {pump }}, \theta_{\text {probe }}\right)$ in terms of the irreducible representations of $\mathrm{D}_{4 \mathrm{~h}}$ point group as:

$$
\begin{gathered}
x^{(3)}\left(\theta_{\text {pump }}, \theta_{\text {probe }}\right)=\frac{1}{2}\left(x_{\mathrm{A}_{19}}^{(3)}+x_{\mathrm{B}_{19}}^{(3)} \cos 2 \theta_{\text {pump }} \cos 2 \theta_{\text {probe }}\right. \\
\left.+x_{\mathrm{B}_{2 g}}^{(3)} \sin 2 \theta_{\text {pump }} \sin 2 \theta_{\text {probe }}\right)
\end{gathered}
$$

where we have defined the symmetry-resolved nonlinear response functions: $X_{\mathrm{A}_{19}}^{(3)}=X_{\text {aaaa }}^{(3)}+X_{\text {bbaa, }}^{(3)}, X_{\mathrm{B}_{19}}^{(3)}=X_{\text {aaaa }}^{(3)}-X_{\text {bbaa }}^{(3)}$ and $X_{\mathrm{B}_{2 g}}^{(3)}=X_{\mathrm{abab}}^{(3)}+X_{\mathrm{abba}}^{(3)}$. and $\theta_{\text {probe/pump }}$ are the angles between the probe/pump polarization vectors and the $a$ axis of the one Fe unit cell. The $A_{1 g}$ is the fully symmetric representation and the $\mathrm{B}_{1 \mathrm{~g}} / \mathrm{B}_{2 \mathrm{~g}}$ representation transform as $x^{2}-y^{2}$ and $x y$, respectively. 

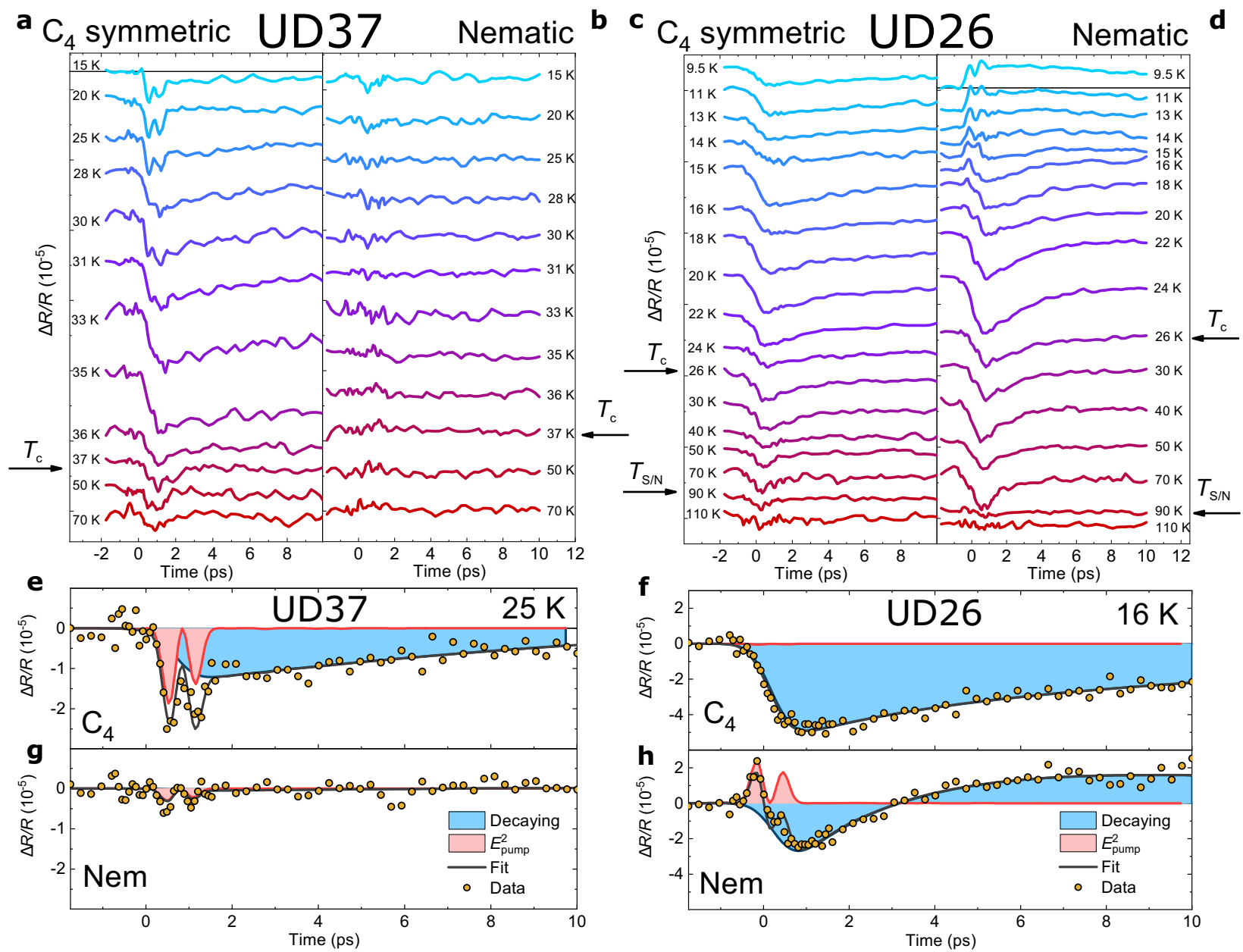

Fig. 2 Temperature and symmetry dependence of the THz response. $\Delta R / R$ against the delay time in the $C_{4}$ symmetric $\left(C_{4}\right)$ and nematic $(\mathrm{Nem})$ channels at various temperatures for $\operatorname{UD} 37(\mathbf{a}, \mathbf{b})$ and $\operatorname{UD} 26(\mathbf{c}, \mathbf{d})$. Fitted curves for the different components of $\Delta R / R$ at $T<T_{\mathrm{c}}$ for UD37 $(\mathbf{e}, \mathbf{g})$ and UD26 (f, h).

The $B_{1 g}$ representation has the same symmetry as the $C_{2}$ symmetric nematic order parameter found in Fe SC. For $\theta_{\text {pump }}=0$, the $A_{1 g}$ and $B_{1 g}$ responses can be accessed using two distinct probe polarization orientations. Indeed making use of Eq. (1) we can write:

$$
\begin{gathered}
\frac{\Delta R C_{4}}{R}=\frac{\Delta R_{\mathrm{a}}}{R_{\mathrm{a}}}+\frac{\Delta R_{\mathrm{b}}}{R_{\mathrm{b}}} \propto \operatorname{ReX}_{\mathrm{A}_{1 \mathrm{~g}}}^{(3)} \\
\frac{\Delta R^{\text {nem }}}{R}=\frac{\Delta R_{\mathrm{a}}}{R_{\mathrm{a}}}-\frac{\Delta R_{\mathrm{b}}}{R_{\mathrm{b}}} \propto \operatorname{ReX}_{\mathrm{B}_{1 \mathrm{~g}}}^{(3)}
\end{gathered}
$$

where $R_{i}(i=\mathrm{a}, \mathrm{b})$ denotes the reflectivity for a probe polarization along the Fe-Fe axes $(a, b)$ of Fig. $1 b$ and for a fixed pump polarization along the $a$ axis. Here, we have taken $R_{\mathrm{a}}=R_{\mathrm{b}}\left(\mathrm{C}_{4}\right.$ tetragonal symmetry). Since the notations $B_{1 g}$ and $A_{1 g}$ are no longer valid in the $C_{2}$ symmetric orthorhombic phase, we will adopt the notation " $\mathrm{C}_{4}$ " for $\mathrm{C}_{4}$ symmetric and "nem" for nematic (or $\mathrm{C}_{2}$ symmetric) when discussing the results below.

\section{Response in the $\mathbf{C}_{\mathbf{4}}$ symmetric superconducting state}

We start by discussing the $\mathrm{C}_{4}$ symmetric and nematic components of the TPOP signal of the UD37 crystal for which only superconductivity is present. Figure $2 \mathrm{a}$, b show the transient reflectivity obtained for both $\mathrm{C}_{4}$ symmetric and nematic components in the UD37 crystal and at various temperatures ranging from $15 \mathrm{~K}$ to $70 \mathrm{~K}$. The decaying part of the $\Delta R / R$ signal shows a strong increase in the $C_{4}$ symmetric channel across $T_{c}$ indicating the superconducting transition (Inset of Fig. 3a). The instantaneous Kerr component is only observed below $T_{\mathrm{c}}$, confirming it is linked to the onset of superconductivity. On the other hand, in the nematic channel no significant changes appear in the transient reflectivity at all temperatures. Using the fitting procedure displayed in Fig. 2e, g, we can obtain the temperature dependencies of the instantaneous and decaying components of $\frac{\Delta R^{C}}{R}$ and $\frac{\Delta R^{n e m}}{R}$ (Fig. 3a). The decaying component displays a sharp extremum around $T_{\mathrm{c}}$ and is assigned to the dynamical relaxation of quasiparticles in the SC state (see Supplementary Note 2). The instantaneous component, attributed to the $\mathrm{THz}$ Kerr effect, shows a strong enhancement below $T_{\mathrm{c}}$. The absence of the instantaneous Kerr component in the nematic channel argues in favor of a contribution arising from the Higgs excitation. Indeed, as shown in the case of $\mathrm{Bi}_{2} \mathrm{Sr}_{2} \mathrm{CaCu}_{2} \mathrm{O}_{8+x}$ cuprates CDF are expected to contribute to all symmetry channels whereas the Higgs contribution is only active in the fully symmetric, i.e., $\mathrm{C}_{4}$ symmetric for a tetragonal crystal, channel ${ }^{35,36,40,42,50}$. Interestingly, Raman scattering spectra on the same crystal in the SC state are dominated by the $B_{1 g}$ channel $^{11}$ (see Supplementary Fig. 1), indicating they mostly probe CDF contributions in stark contrast with the $\mathrm{THz}$ Kerr signal. We note that the respective weight between Higgs and CDF contributions to third-order nonlinear susceptibilities has been a subject of a debate since BCS calculations indicate dominant CDF contributions for a clean superconductor $^{32}$. However, there is now an emerging consensus that disorder significantly boosts the Higgs contribution, thus giving a rationale to the dominance of the Higgs contribution 


\section{4}
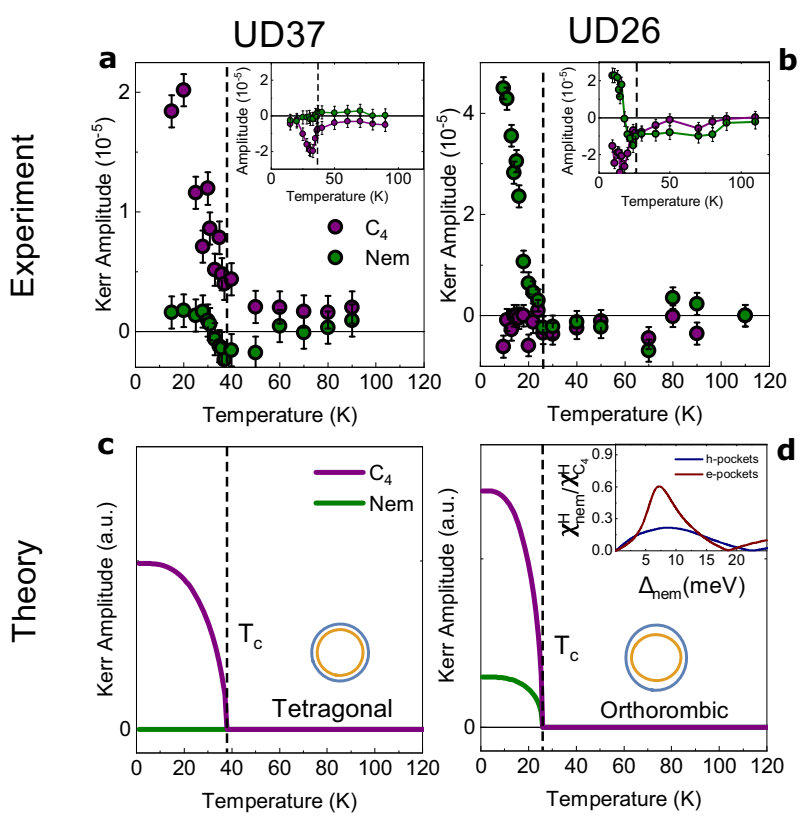

Fig. 3 Instantaneous Kerr response. Temperature dependencies of the amplitude of the fitted instantaneous Kerr signals in the $C_{4}$ symmetric $\left(C_{4}\right)$ and nematic (nem) channels for UD37 (a) and UD26 (b). Insets: Temperature dependencies of the amplitude of the fitted SC decaying signals. Calculated temperature dependence of Higgs contribution of the hole pockets to the instantaneous Kerr signals in the $C_{4}$ symmetric $\left(C_{4}\right)$ and nematic (nem) channels for a tetragonal (c) and orthorombic (d) symmetry of the electronic dispersion relation. The shape of the Fermi surfaces of our model is represented in blue and yellow. Inset: Ratio of the nematic and $C_{4}$ symmetric components of the Higgs response as a function of the nematic order parameter for the hole (blue) and electron pockets (red). Error bars are defined as the standard deviation.

observed in THz Kerr and THG experiments in all superconductors

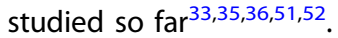

\section{Response in the $\boldsymbol{C}_{\mathbf{2}}$ symmetric superconducting state}

Having discussed the simple case of the $C_{4}$ symmetric superconductor case, let us now turn to the sample with a lower doping level, UD26 which display a $C_{2}$ symmetric SC phase with both nematic and SC orders. Figure $2 c$, d show the transient reflectivity obtained for both channels and at various temperatures ranging from $9.5 \mathrm{~K}$ to $110 \mathrm{~K}$. Above $T_{\mathrm{c}}$ in contrast to UD37, both $C_{4}$ symmetric and nematic components show a change in the transient reflectivity below $T_{\mathrm{S} / \mathrm{N}} \sim 90 \mathrm{~K}$ indicating the transition to the $C_{2}$ symmetric nematic phase. The onset of a decaying signal in the nematic channel is consistent with optical pump optical probe measurements on $\mathrm{BaFe}_{2}\left(\mathrm{As}_{1-x} \mathrm{P}_{x}\right)_{2}$ which reported a similar strongly anisotropic signal below $T_{\mathrm{S} / \mathrm{N}}{ }^{53}$. In principle, a mixture of $C_{2}$ domains of different orientation would average out the nematic component of our signal. The fact that we observe a significant non-zero $\frac{\Delta R^{\text {nem }}}{R}$ shows that one domain orientation prevails under our $250 \mu \mathrm{m}$ laser spot. We attribute this relatively large domain size to residual strains on the sample due to sample mounting which act as symmetric breaking field and align the nematic domains.

Below $T_{\mathrm{c}}$ an instantaneous Kerr component of $\Delta R / R$ that follows the squared THz-pump E-field is also identified, with however a striking difference compared to UD37. Indeed, while it is essentially absent in $\frac{\Delta R}{R}{ }^{C}$, the instantaneous Kerr signal shows a strong enhancement below $T_{c}$ in the $\frac{\Delta R^{\text {nem }}}{R}$ channel. Using the fitting procedure displayed in Fig. $2 \mathrm{f}, \mathrm{h}$, we obtained the amplitude of the instantaneous Kerr and decaying components (see Fig. 3b and Supplementary Fig. 2). Interestingly, the channel dependencies of the instantaneous Kerr and decaying signal are distinct: while the instantaneous Kerr signal is fully nematic with no $C_{4}$ symmetric component within our experimental accuracy, the decaying signal is present in both channels with similar amplitudes at all temperatures.

\section{Origin of the nematic response}

The complete switch from $C_{4}$ symmetric to nematic channel of the instantaneous Kerr signal when going from the $\mathrm{C}_{4} \mathrm{SC}$ phase to the $\mathrm{C}_{2} \mathrm{SC}$ phase is the central finding of the present work. It indicates an unanticipated and profound impact of the $C_{4}$ symmetry breaking on the $\mathrm{THz}$ Kerr nonlinear optical signal of the SC state. We now explore different scenarios to explain this phenomena. First since the structural transition from tetragonal to orthorhombic involves a mixing of the $A_{1 g}$ and $B_{1 g}$ symmetry into the $A_{g}$ symmetry, we naturally expect some mixing of the $\frac{\Delta R}{R}$ symmetry components due to the anisotropy of the optical constants. Based on optical measurements on detwinned $\mathrm{BaFe}_{2} \mathrm{As}_{2}$ samples ${ }^{54}$ (see Supplementary Note 4), we determined quantitatively how the two symmetries are mixed from the calculation of the $\frac{1}{R} \delta R$ prefactor in Eq. (1). We found at most a $25 \%$ anisotropy with respect to the $a$ and $b$ axes. As expected this anisotropy causes a non-zero nematic component. However, it leads to a $\frac{\Delta R^{\text {nem }}}{R}$ signal of at most $10 \%$ of the $\frac{\Delta R}{R} C_{4}$ signal, and therefore plays a marginal role in the $C_{4}$ symmetric to nematic transition observed in the THz Kerr signal.

Having ruled out a simple effect of anisotropic linear optical constant, we are left with the properties of the nonlinear response $x^{(3)}$ itself. In the $C_{2}$ phase, the anisotropy of the electronic dispersion relation will also induce a non-zero component of the Higgs mode response in the nematic channel ${ }^{37}$. We evaluated the activation of the Higgs response in the nematic channel by calculating the third-order nonlinear Higgs response (see Fig. 3c, d for the contribution of the hole pockets) using a three pocket model ( 2 hole-like and 1 electron-like) and an s-wave superconducting gap (see Supplementary Note 3 and 4). As expected, in the $C_{4}$ phase the Higgs response appears below $T_{c}$ only in the $C_{4}$ symmetric channel in agreement with our observations in the UD37 sample. In the $C_{2}$ phase however, the distorted Fermi pockets due to finite nematic order parameter activate the Higgs mode in the nematic channel as observed experimentally. The activation grows with the nematic splitting energy $\Delta_{\text {nem, }}$ but quickly saturates and decreases (see inset of Fig. 3d). We found that for any realistic nematic splitting energy and band parameters, the nematic response of either hole or electron pockets is at most $60 \%$ of the $C_{4}$ symmetric response, thus failing to explain the experimental observation. We note that a dominant contribution from CDF to the $\mathrm{THz}$ Kerr signal would be inconsistent with both the fully $\mathrm{C}_{4}$ symmetric Kerr signal observed in UD37 and the fully nematic Kerr signal observed in UD26 (see Supplementary Note 5 for an evaluation of the CDF contribution).

\section{DISCUSSION}

From the above discussion, it appears that the strong dominance of the THz Kerr signal in the nematic channel of UD26 cannot be explained simply by the effect of the anisotropy of the optical constant or the electronic structure on the Higgs signal. We are thus left with more speculative scenarios. First, we discuss the possibility of an exotic SC order parameter in the $C_{2}$ phase. We note that an SC order parameter with lower symmetry like $d$ wave will not by itself activate a Higgs Kerr signal in non-fully symmetric channels as demonstrated in the case of cuprates ${ }^{37,42}$, so that our observations cannot be easily linked to a change in SC gap symmetry at least for a single band superconductor. However, in multi-orbital systems like Fe SC it is possible that the internal 
structure of Cooper pairs in orbital space profoundly affects the anisotropy of the Kerr Higgs signal. An intriguing possibility is the recent proposal of an orbital-selective SC state in the $C_{2}$ phase of $\mathrm{FeSe}^{13}$. Whether such state would by itself yield a Higgs signal in the nematic channel only is unclear and deserves further theoretical investigations.

Another possibility is that the THz Kerr signal arises from an SC collective mode which couples to the nematic order parameter. In $\mathrm{Ba}_{1-x} \mathrm{~K}_{x} \mathrm{Fe}_{2} \mathrm{As}_{2} s$ and $d$-wave pairing channels are close competitors, potentially giving rise to a BS mode in the nematic $d$ wave channel ${ }^{10,55,56}$. Several spectral features of the Raman spectrum of $\mathrm{Ba}_{1-x} \mathrm{~K}_{x} \mathrm{Fe}_{2} \mathrm{As}_{2}$ have indeed been interpreted as $\mathrm{BS}$ modes, consistent with theoretical evaluations of pairing instabilities in hole-doped $\mathrm{BaFe}_{2} \mathrm{As}_{2}{ }^{10,11,57,58}$ (see also Supplementary Note 1 for a discussion of the Raman spectra in the SC state). Recently, Muller et al. have argued that in the $C_{2}$ phase the BS mode will couple to the amplitude mode of the nematic order parameter, giving rise to a single coupled nematic-BS mode below the Higgs mode energy due to the appearance of a strongly mixed $s+d$ SC state ${ }^{24}$. The stronger decaying signal in the UD26 sample compared to the UD37 sample well-below $T_{c}$ supports the idea of an increased anisotropy of the SC gap in the $C_{2}$ SC phase in agreement with a significant $d$ wave admixture. Interestingly, for parameters close to the critical point where the nematic phase terminates Muller et al. found that the coupled nematic-BS mode may become dominant over the Higgs mode in the short-time dynamics after a quench ${ }^{24}$. Furthermore, we note that the BS mode has the nematic $B_{1 \mathrm{~g}}$ symmetry and will naturally give rise to a signal in the nematic channel $^{59}$. A computation of the third-order nonlinear susceptibility taking into account both $s$ and $d$ pairing channels in the presence of a finite nematic order parameter is desirable to further assess this scenario.

In conclusion, we have studied the impact of nematicity on the SC collective modes in $\mathrm{Ba}_{1-x} \mathrm{~K}_{x} \mathrm{Fe}_{2} \mathrm{As}_{2}$ via $\mathrm{THz}$ pump optical probe measurements. In the absence of nematicity we observe an instantaneous behavior of the optical reflectivity which we assign to a THz Kerr coupling to the Higgs mode. In the coexisting nematic + SC phase we observe a drastic change in the polarization dependence of the THz Kerr signal from purely $C_{4}$ symmetric to purely nematic. The change cannot be accounted by the anisotropy of the electronic properties and indicates the emergence of an SC collective mode which couples strongly to the nematic order parameter. The exact identification of this mode requires further investigation, but we suggest the BS mode connecting nearly degenerate $s$ and $d$ wave pairing ground states as a likely candidate.

\section{METHODS}

\section{Samples}

The two single crystals of $\mathrm{Ba}_{1-x} \mathrm{~K}_{x} \mathrm{Fe}_{2} \mathrm{As}_{2}$ with $T_{\mathrm{c}}=26 \mathrm{~K}(\mathrm{UD} 26, x \sim 0.23)$ and $T_{\mathrm{c}}=37 \mathrm{~K}$ (UD37, $\left.x \sim 0.28\right)$ were characterized by SQUID magnetometry, wavelength dispersing spectroscopy and Raman scattering measurements. The samples are square-shaped with sides of $\sim 5 \mathrm{~mm}$. The crystal orientations of both samples were confirmed by polarization-resolved Raman spectroscopy measurements.

\section{Terahertz pump-optical reflectivity probe (TPOP)}

Strong single cycle $\mathrm{THz}$ pump pulses $(0.3-1 \mathrm{THz})$ with electric field reaching up to $350 \mathrm{kV} / \mathrm{cm}$ are generated using optical rectification of $1.5 \mathrm{eV} \mathrm{NIR}$ pulses in a $\mathrm{LiNbO}_{3}$ crystal using the tilted pulse front technique ${ }^{60,61}$. For optical probe measurements $100 \mathrm{fs}$ duration NIR pulses at $1.5 \mathrm{eV}$ are used with a fluence of $10-100 \mathrm{~nJ} / \mathrm{cm}^{2}$ and a spot size of $250 \mu \mathrm{m}$ in diameter. The repetition rate of the NIR laser is $1 \mathrm{kHz}$.

\section{DATA AVAILABILITY}

The data that support the findings of this study are available from the corresponding authors (R.G., Y.G., and R.S.) upon reasonable request.

\section{CODE AVAILABILITY}

All the numerical codes that support the findings of this study are available from the corresponding authors (R.G., Y.G., and R.S.) upon reasonable request.

Received: 16 June 2021; Accepted: 1 December 2021; Published online: 13 January 2022

\section{REFERENCES}

1. Fradkin, E., Kivelson, S. A. \& Tranquada, J. M. Colloquium: theory of intertwined orders in high temperature superconductors. Rev. Mod. Phys. 87, 457-482 (2015)

2. Fernandes, R. M., Chubukov, A. V. \& Schmalian, J. What drives nematic order in iron-based superconductors? Nat. Phys. 10, 97-104 (2014).

3. Yonezawa, $\mathrm{S}$. et al. Thermodynamic evidence for nematic superconductivity in $\mathrm{Cu}_{x} \mathrm{Bi}_{2} \mathrm{Se}_{3}$. Nat. Phys. 13, 123-126 (2017).

4. Kerelsky, A. et al. Maximized electron interactions at the magic angle in twisted bilayer graphene. Nature 572, 95-100 (2019).

5. Cao, Y. et al. Nematicity and competing orders in superconducting magic-angle graphene. Science 372, 264-271 (2021).

6. Canfield, P. C. \& Bud'ko, S. L. FeAs-based superconductivity: a case study of the effects of transition metal doping on $\mathrm{BaFe}_{2} \mathrm{As}_{2}$. Annu. Rev. Condens. Matter Phys. 1, 27-50 (2010).

7. Chu, J.-H., Kuo, H.-H., Analytis, J. G. \& Fisher, I. R. Divergent nematic susceptibility in an iron arsenide superconductor. Science 337, 710-712 (2012).

8. Gallais, Y. et al. Observation of incipient charge nematicity in $\mathrm{Ba}\left(\mathrm{Fe}_{1-x} \mathrm{Co}_{x}\right)_{2} \mathrm{As}_{2}$. Phys. Rev. Lett. 111, 267001 (2013).

9. Böhmer, A. E. et al. Superconductivity-induced re-entrance of the orthorhombic distortion in $\mathrm{Ba}_{1-x} \mathrm{~K}_{x} \mathrm{Fe}_{2} \mathrm{As}_{2}$. Nat. Commun. 6, 7911 (2015).

10. Graser, S., Maier, T. A., Hirschfeld, P. J. \& Scalapino, D. J. Near-degeneracy of several pairing channels in multiorbital models for the Fe pnictides. N. J. Phys. 11, 025016 (2009).

11. Böhm, T. et al. Microscopic origin of Cooper pairing in the iron-based superconductor $\mathrm{Ba}_{1-x} \mathrm{~K}_{x} \mathrm{Fe}_{2} \mathrm{As}_{2}$. npj Quantum Mater. 3, 48 (2018).

12. Fernandes, R. M. \& Millis, A. J. Nematicity as a probe of superconducting pairing in iron-based superconductors. Phys. Rev. Lett. 111, 127001 (2013).

13. Sprau, P. O. et al. Discovery of orbital-selective Cooper pairing in FeSe. Science 357, 75-80 (2017).

14. Littlewood, P. B. \& Varma, C. M. Amplitude collective modes in superconductors and their coupling to charge-density waves. Phys. Rev. B 26, 4883-4893 (1982).

15. Moor, A., Volkov, P. A., Volkov, A. F. \& Efetov, K. B. Dynamics of order parameters near stationary states in superconductors with a charge-density wave. Phys. Rev. $B$ 90, 024511 (2014).

16. Fu, W., Hung, L.-Y. \& Sachdev, S. Quantum quenches and competing orders. Phys. Rev. B 90, 024506 (2014).

17. Raines, Z. M., Stanev, V. G. \& Galitski, V. M. Hybridization of Higgs modes in a bond-density-wave state in cuprates. Phys. Rev. B 92, 184511 (2015).

18. Dzero, M., Khodas, M. \& Levchenko, A. Amplitude modes and dynamic coexistence of competing orders in multicomponent superconductors. Phys. Rev. B 91 214505 (2015).

19. Gallais, Y., Paul, I., Chauvière, L. \& Schmalian, J. Nematic Resonance in the Raman Response of Iron-Based Superconductors. Phys. Rev. Lett. 116, 017001 (2016).

20. Soto-Garrido, R., Wang, Y., Fradkin, E. \& Cooper, S. L. Higgs modes in the pair density wave superconducting state. Phys. Rev. B 95, 214502 (2017).

21. Sentef, M. A., Tokuno, A., Georges, A. \& Kollath, C. Theory of laser-controlled competing superconducting and charge orders. Phys. Rev. Lett. 118, 087002 (2017).

22. Morice, C., Chakraborty, D. \& Pépin, C. Collective mode in the SU(2) theory of cuprates. Phys. Rev. B 98, 224514 (2018).

23. Müller, M. A., Volkov, P. A., Paul, I. \& Eremin, I. M. Collective modes in pumped unconventional superconductors with competing ground states. Phys. Rev. B 100 140501(R) (2019).

24. Müller, M. A., Volkov, P. A., Paul, I. \& Eremin, I. M. Interplay between nematicity and Bardasis-Schrieffer modes in the short-time dynamics of unconventional superconductors. Phys. Rev. B 103, 024519 (2021).

25. Pekker, D. \& Varma, C. Amplitude/Higgs modes in condensed matter physics. Annu. Rev. Condens. Matter Phys. 6, 269-297 (2015). 
26. Shimano, R. \& Tsuji, N. Higgs mode in superconductors. Annu. Rev. Condens. Matter Phys. 11, 103-124 (2020).

27. Sooryakumar, R. \& Klein, M. V. Raman scattering by superconducting-gap excitations and their coupling to charge-density waves. Phys. Rev. Lett. 45, 660-662 (1980).

28. Méasson, M.-A. et al. Amplitude Higgs mode in the $2 \mathrm{H}-\mathrm{NbSe}_{2}$ superconductor. Phys. Rev. B 89, 060503(R) (2014).

29. Grasset, R. et al. Higgs-mode radiance and charge-density-wave order in 2H-NbSe 2 . Phys. Rev. B 97, 094502 (2018).

30. Grasset, R. et al. Pressure-induced collapse of the charge density wave and Higgs mode visibility in $2 \mathrm{H}-\mathrm{TaS}_{2}$. Phys. Rev. Lett. 122, 127001 (2019).

31. Tsuji, N. \& Aoki, H. Theory of Anderson pseudospin resonance with Higgs mode in superconductors. Phys. Rev. B 92, 064508 (2015).

32. Cea, T., Castellani, C. \& Benfatto, L. Nonlinear optical effects and third-harmonic generation in superconductors: cooper pairs versus Higgs mode contribution. Phys. Rev. B 93, 180507(R) (2016).

33. Murotani, Y. \& Shimano, R. Nonlinear optical response of collective modes in multiband superconductors assisted by nonmagnetic impurities. Phys. Rev. B 99, 224510 (2019).

34. Udina, M., Cea, T. \& Benfatto, L. Theory of coherent-oscillations generation in terahertz pump-probe spectroscopy: From phonons to electronic collective modes. Phys. Rev. B 100, 165131 (2019).

35. Tsuji, N. \& Nomura, Y. Higgs-mode resonance in third harmonic generation in $\mathrm{NbN}$ superconductors: Multiband electron-phonon coupling, impurity scattering, and polarization-angle dependence. Phys. Rev. Res. 2, 043029 (2020).

36. Seibold, G., Udina, M., Castellani, C. \& Benfatto, L. Third harmonic generation from collective modes in disordered superconductors. Phys. Rev. B 103, 014512 (2021).

37. Schwarz, L. \& Manske, D. Theory of driven Higgs oscillations and third-harmonic generation in unconventional superconductors. Phys. Rev. B 101, 184519 (2020).

38. Matsunaga, R. et al. Higgs amplitude mode in the bcs superconductors $\mathrm{Nb}_{1-x} \mathrm{Ti}_{x} \mathrm{~N}$ induced by terahertz pulse excitation. Phys. Rev. Lett. 111, 057002 (2013).

39. Matsunaga, R. et al. Light-induced collective pseudospin precession resonating with Higgs mode in a superconductor. Science 345, 1145-1149 (2014).

40. Matsunaga, R. et al. Polarization-resolved terahertz third-harmonic generation in a single-crystal superconductor $\mathrm{NbN}$ : Dominance of the Higgs mode beyond the BCS approximation. Phys. Rev. B 96, 020505(R) (2017).

41. Nakamura, $\mathrm{S}$. et al. Infrared activation of the Higgs mode by supercurrent injection in superconducting NbN. Phys. Rev. Lett. 122, 257001 (2019).

42. Katsumi, K. et al. Higgs mode in the $d$-wave superconductor $\mathrm{Bi}_{2} \mathrm{Sr}_{2} \mathrm{CaCu}_{2} \mathrm{O}_{8+x}$ driven by an intense terahertz pulse. Phys. Rev. Lett. 120, 117001 (2018).

43. Chu, H. et al. Phase-resolved Higgs response in superconducting cuprates. Nat. Commun. 11, 1793 (2020).

44. Yang, X. et al. Lightwave-driven gapless superconductivity and forbidden quantum beats by terahertz symmetry breaking. Nat. Photonics 13, 707-713 (2019).

45. Vaswani, C. et al. Light quantum control of persisting Higgs modes in iron-based superconductors. Nat. Commun. 12, 258 (2021).

46. Hoffmann, M. C., Brandt, N. C., Hwang, H. Y., Yeh, K.-L. \& Nelson, K. A. Terahertz Kerr effect. Appl. Phys. Lett. 95, 231105 (2009).

47. Yang, X. et al. Lightwave-driven gapless superconductivity and forbidden quantum beats by terahertz symmetry breaking. Nat. Photonics 13, 707-713 (2019).

48. Boyd, R. W. Nonlinear Optics 3 (Academic Press, Inc., 2008).

49. Paul, I. Nonlinear terahertz electro-optical responses in metals. Preprint at http:// arxiv.org/abs/2101.04136 (2021).

50. Cea, T., Barone, P., Castellani, C. \& Benfatto, L. Polarization dependence of the third-harmonic generation in multiband superconductors. Phys. Rev. B 97, 094516 (2018).

51. Silaev, M. Nonlinear electromagnetic response and Higgs-mode excitation in BCS superconductors with impurities. Phys. Rev. B 99, 224511 (2019).

52. Jujo, T. Quasiclassical theory on third-harmonic generation in conventional superconductors with paramagnetic impurities. J. Phys. Soc. Jpn. 87, 024704 (2018).

53. Thewalt, E. et al. Imaging anomalous nematic order and strain in optimally doped $\mathrm{BaFe}_{2}(\mathrm{As}, \mathrm{P})_{2}$. Phys. Rev. Lett. 121, 027001 (2018).

54. Nakajima, M. et al. Unprecedented anisotropic metallic state in undoped iron arsenide $\mathrm{BaFe}_{2} \mathrm{As}_{2}$ revealed by optical spectroscopy. Proc. Natl Acad. Sci. USA 108, 12238 (2011).
55. Bardasis, A. \& Schrieffer, J. R. Excitons and plasmons in superconductors. Phys. Rev. 121, 1050-1062 (1961).

56. Barlas, Y. \& Varma, C. M. Amplitude or Higgs modes in d-wave superconductors. Phys. Rev. B 87, 054503 (2013).

57. Maiti, S. \& Hirschfeld, P. J. Collective modes in superconductors with competing sand d-wave interactions. Phys. Rev. B 92, 094506 (2015).

58. Maiti, S., Maier, T. A., Böhm, T., Hackl, R. \& Hirschfeld, P. J. Probing the pairing interaction and multiple Bardasis-Schrieffer modes using Raman spectroscopy. Phys. Rev. Lett. 117, 257001 (2016).

59. Müller, M. A. \& Eremin, I. M. Signatures of Bardasis-Schrieffer mode excitation in third-harmonic generated currents. Phys. Rev. B 104, 144508 (2021).

60. Hebling, J., Yeh, K.-L., Hoffmann, M. C., Bartal, B. \& Nelson, K. A. Generation of high-power terahertz pulses by tilted-pulse-front excitation and their application possibilities. J. Opt. Soc. Am. B 25, B6-B19 (2008).

61. Watanabe, S., Minami, N. \& Shimano, R. Intense terahertz pulse induced exciton generation in carbon nanotubes. Opt. Express 19, 1528-1538 (2011).

\section{ACKNOWLEDGEMENTS}

The authors would like to thank M. Müller, I. Eremin, R. Lobo, I. Paul, and L. Benfatto for fruitful discussions. R.G. and Y.G. acknowledge the support from the Japan Society for the Promotion of Science. This work was partly supported by JST CREST Grant No. JPMJCR19T3, Japan.

\section{AUTHOR CONTRIBUTIONS}

The samples were grown by H.-H.W. and X.-H.C.; R.G. and K.K. performed THz pump optical probe experiments; P.M. performed Raman experiments; R.G. and Y.G. performed the calculations and analyzed data; Y.G. and R.S. supervised the project; all authors discussed the results and wrote the manuscript.

\section{COMPETING INTERESTS}

The authors declare no competing interests.

\section{ADDITIONAL INFORMATION}

Supplementary information The online version contains supplementary material available at https://doi.org/10.1038/s41535-021-00411-9.

Correspondence and requests for materials should be addressed to Romain Grasset, Yann Gallais or Ryo Shimano.

Reprints and permission information is available at http://www.nature.com/ reprints

Publisher's note Springer Nature remains neutral with regard to jurisdictional claims in published maps and institutional affiliations.

Open Access This article is licensed under a Creative Commons Attribution 4.0 International License, which permits use, sharing, adaptation, distribution and reproduction in any medium or format, as long as you give appropriate credit to the original author(s) and the source, provide a link to the Creative Commons license, and indicate if changes were made. The images or other third party material in this article are included in the article's Creative Commons license, unless indicated otherwise in a credit line to the material. If material is not included in the article's Creative Commons license and your intended use is not permitted by statutory regulation or exceeds the permitted use, you will need to obtain permission directly from the copyright holder. To view a copy of this license, visit http://creativecommons. org/licenses/by/4.0/.

(c) The Author(s) 2022 